\section{Spurious correlations in research on ability tilt}

\author{
Kimmo Sorjonen ${ }^{1}$, Gustav Nilsonne ${ }^{1,2}$, \\ Michael Ingre ${ }^{2,3}, \&$ Bo Melin ${ }^{1}$
}

${ }^{1}$ Department of Clinical Neuroscience, Karolinska Institutet, Stockholm, Sweden

2 Department of Psychology, Stockholm University, Stockholm, Sweden

${ }^{3}$ Institute for Globally Distributed Open Research and Education (IGDORE), Stockholm, Sweden

Abstract

Ability tilt refers to a within-individual difference between two abilities (X-Y), e.g. differences between tech, verbal and math abilities. Studies have found associations between ability tilts and their constituent abilities (X or Y). Here we investigate whether such associations may be spurious due to the non-independence of the two measures. Using data from the 1997 National Longitudinal Survey of Youth (NLSY), we find that associations between ability and ability tilt may simply be due to more positive associations between two measures of the same or similar abilities compared to two measures of different or dissimilar abilities. This finding calls into question theoretical interpretations that have proposed that ability tilt correlations are due to differential investment of time and effort in one ability at the expense of the other ability.

Key words: ability tilt, differential investment, spurious correlations, statistical expectations

\section{Introduction}

Ability tilt refers to a within-individual difference between two abilities, e.g. higher math ability than verbal ability. There exists a body of research in which ability tilts have been correlated to their constituent abilities. Here we investigate whether such correlations may be spurious because of the non-independence between ability tilt and the constituent abilities.

Analyses of associations involving ability tilts have suggested that math (vs. verbal) tilt, i.e. higher math than verbal ability, correlates positively with math ability and negatively with verbal ability, and vice versa for verbal (vs. math) tilt [1-3], and that tech (vs. academic) tilt correlates negatively with academic ability [4-6]. Studies have also found a positive association between tech (vs. academic) tilt and STEM (science, technology, engineering, and math) criteria (e.g. grades and jobs) [4,6], positive associations between academic (vs. tech) tilt and activity in humanities and verbal fields [4], between math (vs. verbal) tilt and STEM majors, and between verbal (vs. math) tilt and humanities majors [1-3]. A negative association between general intelligence factor $g$ and tech (vs. academic) tilt has also been found [4-6].

Studies of group differences have indicated a tech (vs. academic) and a math (vs. verbal) tilt among men and an academic (vs. tech) and a verbal (vs. math) tilt among women [3,5]. The negative correlation between tech (vs. academic) tilt and academic ability, mentioned above, has been found to be stronger among Blacks compared to Whites [6] and stronger among women compared to men [5].

Coyle [1-6] has proposed that negative correlations between $\mathrm{X}$ (vs. Y) tilts and tests measuring ability $Y$ may be explained by differential investment of time and effort in ability $X$ (e.g. technical ability) at the expense of investment in ability Y (e.g. academic ability). Furthermore, according to Coyle [6], the weaker negative correlation between tech (vs. academic) tilt and academic ability among Whites compared to Blacks may be explained by a generally higher $g$ among Whites in combination with Spearman's law of diminishing returns, which states that associations between cognitive abilities tend to be weaker among those higher in $g$ [7-9].

Pearson [10] introduced the concept of spurious correlation, based on an analysis of correlations between ratio variables with a shared denominator. Pearson noted that even in the absence of an association between the objects under study, such ratios will tend to be correlated for purely arithmetic reasons. Subsequent work has generalized the notion of spurious correlations and applied it to other cases where a variable is correlated to a composite variable containing itself, e.g. [11,12].

If an additive composite variable $\mathrm{X}-\mathrm{Y}$ is correlated to one of its constituent variables $\mathrm{X}$ or $\mathrm{Y}$, the two measures are non-independent. Formally, for two standardized variables, $\mathrm{X}$ and $\mathrm{Y}$, with a normal distribution, mean $=0$ and $\mathrm{SD}=1$, the expected correlation between their difference, $\mathrm{X}-\mathrm{Y}$, and a third variable, $Z$, is given by Eq. 1 . It can be seen that the correlation between $\mathrm{X}-\mathrm{Y}$ and $\mathrm{Z}$ will be stronger if there is a more positive correlation between $\mathrm{X}$ and $\mathrm{Y}$ and if there is a greater difference between the correlations of $\mathrm{X}$ to $\mathrm{Z}$ and $\mathrm{Y}$ to $\mathrm{Z}$.

$$
E\left|r_{x-y, z}\right|=\frac{r_{x z}-r_{y z}}{\sqrt{2\left(1-r_{x y}\right)}} \quad \text { Eq. } 1
$$

If $\mathrm{Z}$ in Eq. 1 is replaced by $\mathrm{X}$ and $\mathrm{Y}$, respectively, we obtain:

$$
\begin{array}{ll}
E\left|r_{x-y, x}\right|=\sqrt{\frac{1-r_{x y}}{2}} & E q .2 \\
E\left|r_{x-y, y}\right|=-\sqrt{\frac{1-r_{x y}}{2}} & E q .3
\end{array}
$$


Hence, unless $\mathrm{X}$ and $\mathrm{Y}$ are perfectly positively correlated, the difference $\mathrm{X}-\mathrm{Y}$ is expected to be positively correlated with $\mathrm{X}$ and equally strongly, but negatively, correlated with Y. Therefore, the X$\mathrm{Y}$ difference is expected to be positively (negatively) correlated with anything that $\mathrm{X}$ is positively (negatively) correlated with and negatively (positively) correlated with whatever $\mathrm{Y}$ is positively (negatively) correlated with.

Eq. 2 and Eq. 3 might explain why studies have found positive correlations between X (vs. Y) tilts and the constituent ability $X$ and negative correlations between $\mathrm{X}$ (vs. Y) tilts and the constituent ability Y. However, our criticism is not restricted to the case where an ability tilt is correlated to one of its constituent measures. If the $\mathrm{X}$ (vs. Y) tilt is correlated with another measure of one of the constituent abilities (X or Y), for example if tech (vs. academic) tilt is correlated with another measure of academic ability than the one used for calculating the tilt, we would use Eq. 1 instead of Eq. 2 or Eq. 3. However, the expected correlation would be similar, as we can assume a more positive correlation between two different measures of the same ability than between two measures of different abilities. The positive association between tech (vs. academic) and math (vs. verbal) tilt and STEM criteria, mentioned above, would be in accordance with Eq. 1 if we were to assume a more positive correlation between technical and math abilities and STEM criteria $\left(r_{x z}\right)$ than between academic and verbal abilities and STEM criteria $\left(r_{y z}\right)$. Similar explanations are possible for other reported associations between ability tilts and college majors and jobs. A negative association between $g$ and tech (vs. academic) tilt could simply be due to a more positive correlation between $g$ and academic ability compared to between $g$ and technical ability.

Differences in association involving ability tilts between groups could be due to differences in the correlations in Eq. 1. For example, the stronger negative correlation between tech (vs. academic) tilt and academic ability among Blacks compared to Whites and among women compared to men could be due to a stronger correlation between technical and academic abilities $\left(r_{x y}\right)$ among Whites and among men compared to Blacks and women.

The objective of the present study was to investigate if reported findings in the ability tilt literature can be explained as spurious correlations.

Method

Although not meant as an exact replication, we followed a very similar procedure as Coyle, e.g. [6], both for acquiring data and for the analyses.

\section{Participants}

Participants were US youth born between 1980 and 1984 in the 1997 National Longitudinal Survey of Youth (NLSY) dataset. The full sample consists of 8984 participants (2335 Blacks, 4665 nonHispanic Whites, and 1984 with other ethnicity; 4385 women and 4599 men), see the tables for the $n$ in each analysis.

\section{Measurements}

In 1997-1998 most of the participants (7008 with complete data) took 12 Armed Services Vocational Aptitude Battery (ASVAB) tests: (1) general science; (2) arithmetic reasoning; (3) word knowledge; (4) paragraph comprehension; (5) numerical operations; (6) coding speed; (7) auto information; (8) shop information; (9) mathematical knowledge; (10) mechanical comprehension; (11) electronics information; (12) assembling objects. Data on the math and verbal subtests of the three college aptitude tests SAT, ACT, and PSAT were available for between 1003 and 1407 participants.

Data on college majors were available for 1987 of the participants. Based on the latest available major, these participants were categorized into those with a STEM major (e.g. computer science, engineering, and mathematics, $n=136$, coded as 1 ) and those with a humanities major (e.g. languages, law, and liberal arts and sciences, $n=1003$, coded as 0 ). The college course map (CCM) codes used for this categorization can be seen in the available script (see link below). Data on at least one occupation between 1997 and 2017 was available for 8843 of the participants. Based on the latest available occupation, these participants were categorized into those with a STEM job (e.g. computer and mathematical occupations, engineering, and physical sciences, $n=309$, coded as 1) and those with a job in the humanities field (e.g. community and social services, legal occupations, and media, $n$ $=709$, coded as 0 ). The census 2002 occupation codes used for this categorization can be seen in the available script (see link below).

\section{Statistical analyses}

Tech ability was calculated as the withinindividual mean on the standardized auto information, electronics information, mechanical comprehension, and shop information ASVAB scores, verbal ability as the mean on the general science, paragraph comprehension, and word knowledge scores, and math ability as the mean on the arithmetic reasoning and mathematical knowledge scores, respectively. Tech (vs. verbal), tech (vs. math), and math (vs. verbal) tilts were calculated as the within-individual differences between these calculated abilities, respectively. The participants' $g$ was estimated as a factor score on the first unrotated factor in an analysis of all 12 ASVAB tests.

The correlations between the tech, verbal, and math abilities, as well as the three tilts, on one hand and the college aptitude test scores, STEM majors, STEM jobs, and $g$, on the other hand, were 
calculated in the full sample as well as separately among Blacks, Whites, women, and men. The expected correlations involving the tilts were calculated with Eq. 1 and a difference between observed and expected tilt-correlations was accepted if the latter was not included in the $95 \%$ confidence interval of the former. In the group comparisons, the differences between the Fisher's $r$ to $z$ transformed correlations [13] were calculated both for the observed and the expected tilt-correlations and the observed difference was accepted to differ from the expected difference if the latter was not included in the $95 \%$ confidence interval of the former. Analyses were conducted with R 4.0.2 statistical software [14] employing the psych package [15]. The used script and data are available at https://osf.io/3swmb/.

\section{Results}

Twenty-three of the 27 analysed correlations between $\mathrm{X}-\mathrm{Y}$ ability tilts and a third variable $\mathrm{Z}$ were statistically significant (zero not included in the confidence interval; Table 1). However, none of these correlations differed significantly from the expected correlation between $\mathrm{X}-\mathrm{Y}$ and $\mathrm{Z}$ according to Eq. 1. As discussed above, and in accordance with Eq. 1, whenever the correlation between $\mathrm{X}$ and $\mathrm{Z}$ is more positive (negative) than the correlation between $\mathrm{Y}$ and $\mathrm{Z}$, the correlation between the $\mathrm{X}-\mathrm{Y}$ tilt and $\mathrm{Z}$ is positive (negative).

Tables 2 and 3 show that observed correlations between X-Y ability tilts and Z differed significantly from the expected correlation more often among Blacks (5 of 27 cases) compared to Whites (0) and more often among women (14) compared to men (2). The observed ability tilt-correlations differed significantly between Blacks and Whites in 9 cases, but differed from the expected differences in only 5 cases. Between women and men, the observed ability tilt-correlations differed significantly in 10 cases while the observed differences differ from the expected in 14 cases. This latter increase from 10 to 14 cases is due to the fact that the observed and expected differences can have different signs. All group differences are limited to cases where $\mathrm{X}=$ tech in the X-Y tilt. In all of the cases when observed differences differed from expected, the observed ability tilt-correlation is stronger than the expected ability tilt-correlation among Blacks and among women, respectively.

Discussion

In the full sample, all of the correlations between $\mathrm{X}-\mathrm{Y}$ tilts and a third variable $\mathrm{Z}$ were consistent with spurious associations due to non-independence. This

Table 1. Correlations between $\mathrm{X}, \mathrm{Y}$, and Z-variables, as well as the observed and expected correlations between the $\mathrm{X}-\mathrm{Y}$ tilt and Z.

\begin{tabular}{|c|c|c|c|c|c|c|c|}
\hline Variables & $n$ & $r_{x y}$ & $r_{x z}$ & $r_{y z}$ & obs. $r_{x-y, z}(95 \% \mathrm{CI})^{1}$ & exp. $r_{x-y, z^{2}}$ & obs. $\neq$ exp. $^{3}$ \\
\hline \multicolumn{8}{|l|}{$\mathrm{x}=$ Tech., $\mathrm{y}=$ Ver. } \\
\hline $\mathrm{z}=\mathrm{SATm}$ & 1188 & 0.72 & 0.49 & 0.60 & $-0.12(-0.18 ;-0.07)$ & -0.14 & No \\
\hline $\mathrm{z}=\mathrm{SATV}$ & 1187 & 0.72 & 0.49 & 0.74 & $-0.32(-0.37 ;-0.26)$ & -0.33 & No \\
\hline $\mathrm{z}=\mathrm{ACTm}$ & 1101 & 0.72 & 0.50 & 0.62 & $-0.15(-0.20 ;-0.09)$ & -0.17 & No \\
\hline $\mathrm{z}=\mathrm{ACT} v$ & 1100 & 0.72 & 0.49 & 0.72 & $-0.29(-0.34 ;-0.23)$ & -0.31 & No \\
\hline $\mathrm{z}=\mathrm{PSATm}$ & 863 & 0.75 & 0.54 & 0.62 & $-0.09(-0.16 ;-0.03)$ & -0.11 & No \\
\hline $\mathrm{z}=$ PSATv & 864 & 0.75 & 0.54 & 0.76 & $-0.29(-0.35 ;-0.23)$ & -0.31 & No \\
\hline $\mathrm{z}=\mathrm{STEMmajor}$ & 996 & 0.76 & 0.12 & 0.03 & $0.13(0.06 ; 0.19)$ & 0.12 & No \\
\hline $\mathrm{z}=\mathrm{STEMjob}$ & 854 & 0.77 & 0.34 & 0.16 & $0.30(0.23 ; 0.36)$ & 0.26 & No \\
\hline $\mathrm{Z}=g$ & 7079 & 0.79 & 0.87 & 0.95 & $-0.12(-0.14 ;-0.10)$ & -0.12 & No \\
\hline \multicolumn{8}{|l|}{$\mathrm{x}=$ Tech., $\mathrm{y}=$ Math } \\
\hline $\mathrm{z}=\mathrm{SATm}$ & 1188 & 0.61 & 0.49 & 0.68 & $-0.19(-0.24 ;-0.13)$ & -0.21 & No \\
\hline $\mathrm{z}=\mathrm{SATV}$ & 1187 & 0.61 & 0.49 & 0.56 & $-0.05(-0.11 ; 0.01)$ & -0.07 & No \\
\hline $\mathrm{z}=\mathrm{ACTm}$ & 1101 & 0.63 & 0.50 & 0.71 & $-0.24(-0.30 ;-0.19)$ & -0.25 & No \\
\hline $\mathrm{z}=\mathrm{ACTV}$ & 1100 & 0.63 & 0.49 & 0.56 & $-0.08(-0.14 ;-0.02)$ & -0.09 & No \\
\hline $\mathrm{z}=\mathrm{PSATm}$ & 863 & 0.64 & 0.54 & 0.70 & $-0.14(-0.21 ;-0.08)$ & -0.19 & No \\
\hline $\mathrm{z}=\mathrm{PSATV}$ & 864 & 0.64 & 0.54 & 0.58 & $-0.01(-0.08 ; 0.05)$ & -0.05 & No \\
\hline $\mathrm{z}=\mathrm{STEMmajor}$ & 996 & 0.64 & 0.12 & 0.11 & $0.02(-0.05 ; 0.08)$ & 0.01 & No \\
\hline $\mathrm{z}=\mathrm{STEMjob}$ & 854 & 0.66 & 0.34 & 0.17 & $0.23(0.16 ; 0.29)$ & 0.20 & No \\
\hline $\mathrm{Z}=g$ & 7079 & 0.70 & 0.87 & 0.92 & $-0.07(-0.09 ;-0.04)$ & -0.07 & No \\
\hline \multicolumn{8}{|l|}{$\mathrm{x}=$ Math, $\mathrm{y}=$ Ver. } \\
\hline $\mathrm{z}=$ SATm & 1197 & 0.76 & 0.68 & 0.59 & $0.11(0.05 ; 0.16)$ & 0.13 & No \\
\hline $\mathrm{z}=\mathrm{SATV}$ & 1196 & 0.76 & 0.56 & 0.74 & $-0.27(-0.32 ;-0.21)$ & -0.25 & No \\
\hline $\mathrm{z}=\mathrm{ACTm}$ & 1103 & 0.79 & 0.71 & 0.62 & $0.16(0.10 ; 0.22)$ & 0.14 & No \\
\hline $\mathrm{z}=\mathrm{ACT} \mathrm{v}$ & 1102 & 0.79 & 0.56 & 0.72 & $-0.22(-0.28 ;-0.17)$ & -0.24 & No \\
\hline $\mathrm{z}=\mathrm{PSATm}$ & 870 & 0.78 & 0.70 & 0.62 & $0.09(0.02 ; 0.16)$ & 0.12 & No \\
\hline $\mathrm{z}=\mathrm{PSATV}$ & 871 & 0.78 & 0.58 & 0.76 & $-0.30(-0.36 ;-0.24)$ & -0.27 & No \\
\hline $\mathrm{z}=$ STEMmajor & 998 & 0.78 & 0.11 & 0.03 & $0.11(0.05 ; 0.17)$ & 0.11 & No \\
\hline $\mathrm{z}=\mathrm{STEMjob}$ & 858 & 0.79 & 0.17 & 0.16 & $0.02(-0.04 ; 0.09)$ & 0.02 & No \\
\hline $\mathrm{z}=g$ & 7113 & 0.84 & 0.92 & 0.95 & $-0.05(-0.07 ;-0.02)$ & -0.05 & No \\
\hline
\end{tabular}




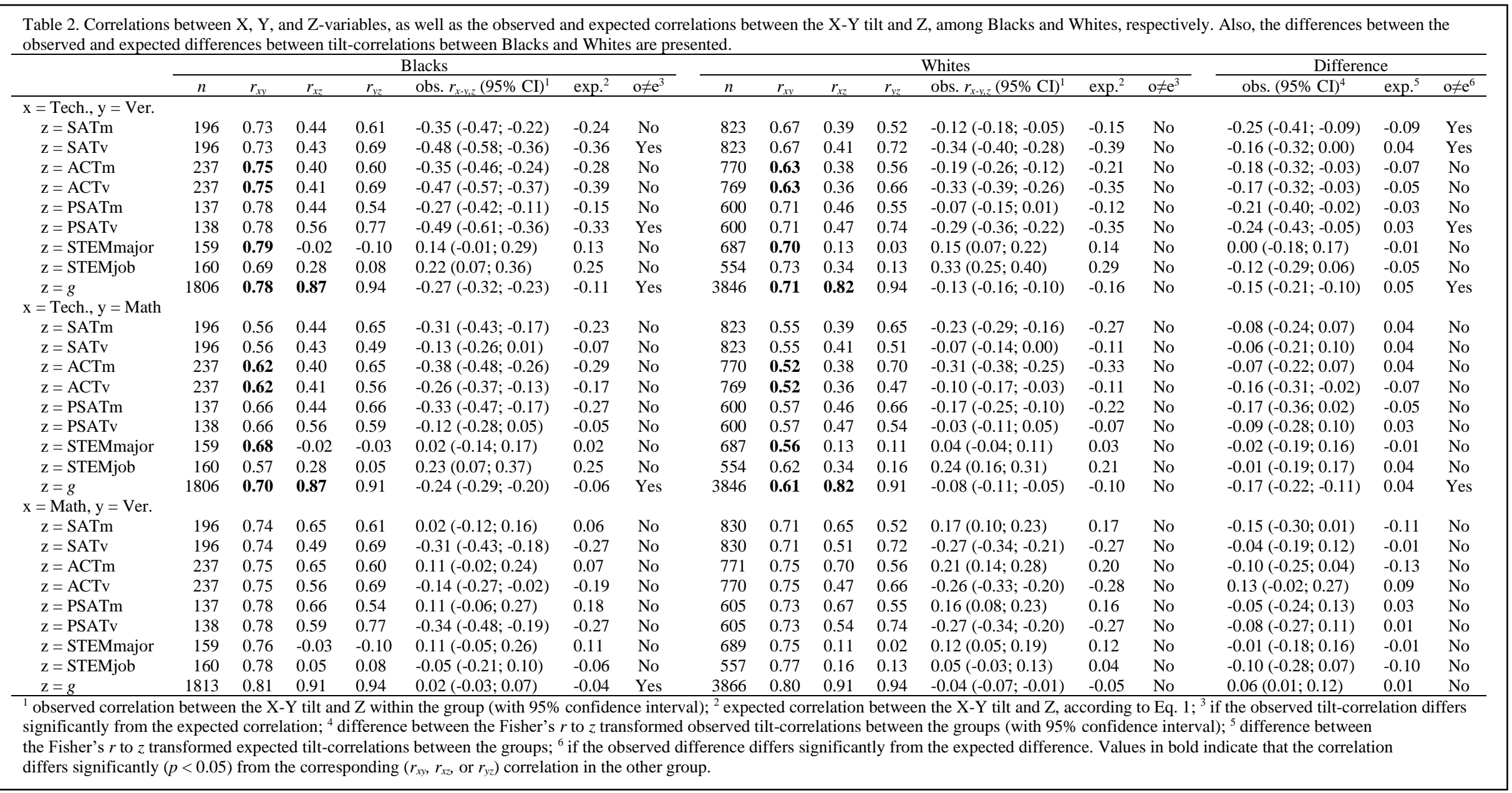




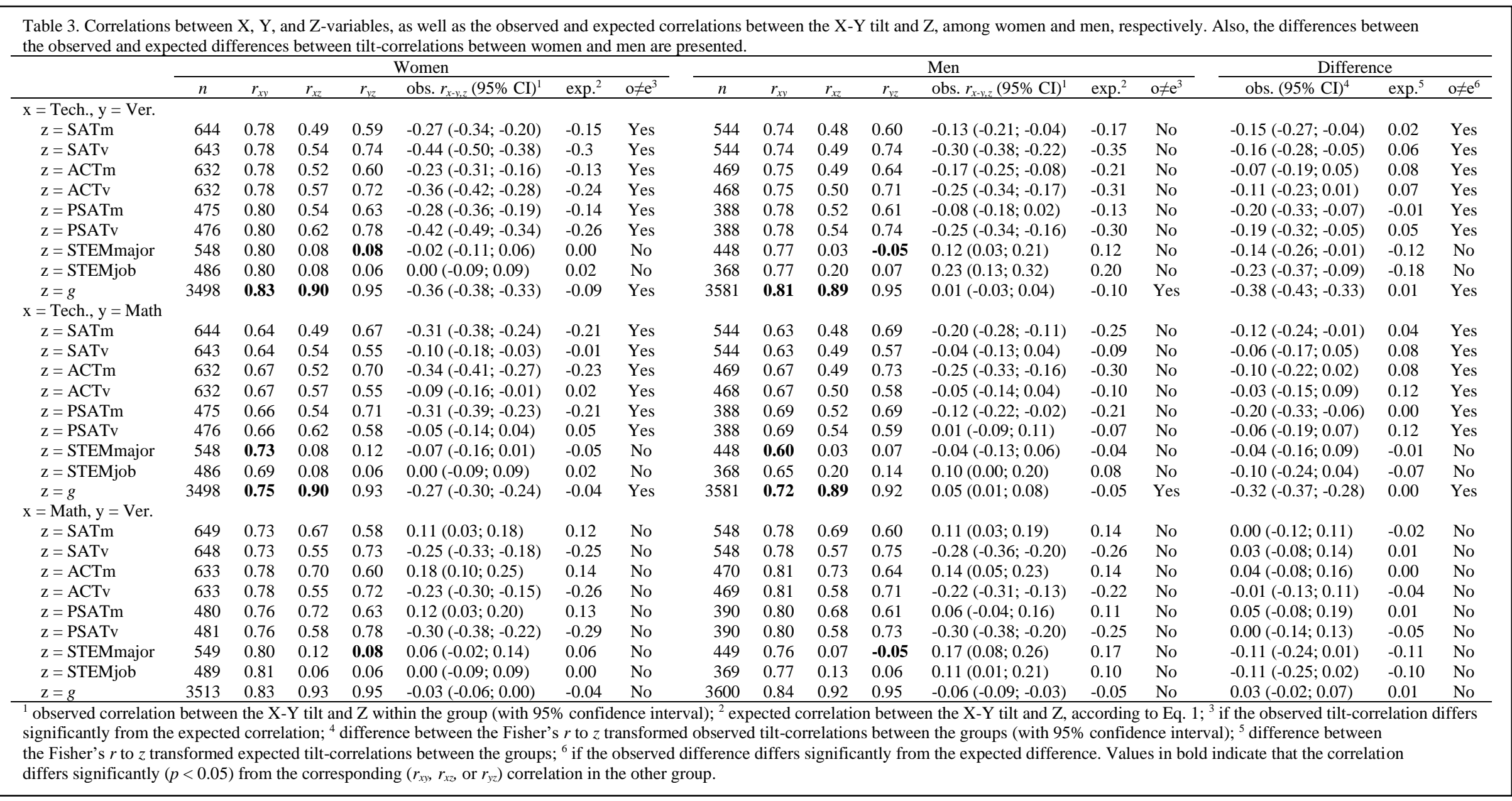


means, for example, that if $\mathrm{Z}$ is measuring the same ability as one of the constituent variables, $\mathrm{X}$ or $\mathrm{Y}$, ability tilt-associations can be explained simply by the tendency that two measures of the same ability correlate more positively compared to two measures of different abilities. Hence, if $\mathrm{Z}$ is a measure of the same ability as X (Y), the correlation between the X$\mathrm{Y}$ tilt and $\mathrm{Z}$ will tend to be positive (negative). This finding calls into question whether investment theories and differential investment can be used, as proposed by Coyle [1-6], to explain such tiltcorrelations.

Some group differences in the strength of the observed correlation between $\mathrm{X}-\mathrm{Y}$ ability tilts and $\mathrm{Z}$ differed significantly from expected differences. In each case this seemed to be due to a stronger than expected tilt-correlation among Blacks and women, respectively. It is an open question why we see this discrepancy between observed and expected tiltcorrelations among Blacks and women in some instances. As mentioned in the introduction, Coyle [6] proposes that the difference in the strength of the tech (vs. academic) tilt and academic ability between Blacks and Whites is due to a difference in $g$ in combination with Spearman's law of diminishing returns [9], which states that the association between cognitive abilities tend to be weaker among those higher in $g$. However, this would not explain why the difference is limited to cases where $\mathrm{X}=$ tech in the $\mathrm{X}-\mathrm{Y}$ ability tilt and not seen for the tilt between math and verbal abilities. Neither would it explain why we see more differences in the strength of tilt-correlations between women and men, again limited to cases where $\mathrm{X}=$ tech. This seems to suggest that the found differences might be due to some peculiarities in the measurement of tech abilities, possibly related to deficient reliability or validity.

The present findings are related to research indicating that some well-established hypotheses about associations involving intelligence are to some degree due to statistical artefacts or confounding. For example, the intelligencecreativity threshold hypothesis claims that there is a positive association between intelligence and creativity, but only up to a certain point (the threshold, which has often been set at IQ = 120), after which a further increase in intelligence is not associated with a further increase in creativity. This hypothesis has received some empirical support, e.g [16], but simulations have indicated that such findings could be due to an effect of disturbance, e.g. low motivation, illness, or linguistic confusion, that varies in degree between research participants and has a simultaneous effect on their performance both on the tests of intelligence and on the tests of creativity [17]. Such an effect of disturbance, and especially if it is negatively skewed, meaning that most participants receive scores close to their true intelligence while a few receive scores well below their true ability, could also have increased the likelihood to get results in accordance with Spearman's law of diminishing returns mentioned above [18].

Another example is the worst performance rule (WPR), which is the name given to the phenomenon that if reaction times, measured over several trials, are sorted from the lowest (= fastest) to the highest (= slowest) value within participants, the negative correlation with intelligence tends to strengthen from the fastest to the slowest trial $[19,20]$. It has been proposed that the WPR phenomenon might be due to a higher risk for lapses in executive control and attention among those with low intelligence which, in turn, might be due to errors in neural processing [21-23]. However, the correlation of sorted scores rule (CSSR [24,25]) suggests that results in accordance with the WPR are exactly what can be expected given the commonly observed negative correlation between intelligence and the within-individual mean and variance in reaction time measured over multiple trials [23,26,27].

A kinship could also be seen between the present study and demonstrations that statistical findings in general are not always indicating what they are commonly believed to indicate. For example, an observed effect of a predictor X (e.g. intelligence) on the difference on an outcome variable $\mathrm{Y}$ (e.g. quality of life) measured at two time-points $\left(\mathrm{Y}_{1}-\mathrm{Y}_{0}\right)$ while adjusting for the baseline value $\left(\mathrm{Y}_{0}\right)$ might be due to an association between $\mathrm{X}$ and $\mathrm{Y}_{0}$ in combination with a less than perfect reliability in the measurement of the outcome $\mathrm{Y}$, rather than due to a true effect of $\mathrm{X}$ on the change in $\mathrm{Y}[28,29]$. It has also been demonstrated that an observed significant effect of a predictor $\mathrm{X}$ on an outcome $\mathrm{Y}$ while adjusting for a possible confounder $\mathrm{Z}$ does not guarantee a true independent association between $X$ and $\mathrm{Y}$, especially in situations with a high degree of true confounding, low reliability in the measurement of $\mathrm{Z}$, high reliability in the measurement of $\mathrm{X}$ and $\mathrm{Y}$, and a large sample size [30,31].

\section{Conclusions}

Associations between ability tilts, e.g. differences between tech and verbal or math abilities, and other variables, e.g. scores on college aptitude tests, can be explained to a large degree as spurious due to the non-independence of the measures, and consequently do not support differential investment as an explanation.

\section{References}

1. Coyle TR. 2016 Ability tilt for whites and blacks: Support for differentiation and investment theories. Intelligence 56, 28-34. (doi:10.1016/j.intell.2016.02.002)

2. Coyle TR, Purcell JM, Snyder AC, Richmond MC. 2014 Ability tilt on the SAT and ACT predicts specific abilities and college majors. Intelligence $\mathbf{4 6}$, 18-24. (doi:10.1016/j.intell.2014.04.008) 
3. Coyle TR, Snyder AC, Richmond MC. 2015 Sex differences in ability tilt: Support for investment theory. Intelligence 50, 209-220. (doi:10.1016/j.intell.2015.04.012)

4. Coyle TR. 2019 Tech tilt predicts jobs, college majors, and specific abilities: Support for investment theories. Intelligence 75, 33-40. (doi:10.1016/j.intell.2019.04.002)

5. Coyle TR. 2020 Sex differences in tech tilt: Support for investment theories. Intelligence 80, 101437. (doi:10.1016/j.intell.2020.101437)

6. Coyle TR. 2021 White-Black differences in tech tilt: Support for Spearman's law and investment theories. Intelligence 84, 101504. (doi:10.1016/j.intell.2020.101504)

7. Blum D, Holling H. 2017 Spearman's law of diminishing returns. A meta-analysis. Intelligence 65, 60-66. (doi:10.1016/j.intell.2017.07.004)

8. Deary IJ, Egan V, Gibson GJ, Austin EJ, Brand CR, Kellaghan T. 1996 Intelligence and the differentiation hypothesis. Intelligence 23, 105-132. (doi:10.1016/S0160-2896(96)90008-2)

9. Spearman C. 1927 The abilities of man. London: Macmillan.

10. Pearson K. 1897 Mathematical contributions to the theory of evolution - On a form of spurious correlation which may arise when indices are used in the measurement of organs. Proc. R. Soc. Lond. 60 , 489-498. (doi:10.1098/rspl.1896.0076)

11. Chayes F. 1949 On ratio correlation in petrography. J. Geol. 57, 239-254. (doi:10.1086/625606)

12. Reed LJ. 1921 On the correlation between any two functions and its application to the general case of spurious correlation. J. Wash. Acad. Sci. 11, 449455.

13. Cohen J, Cohen P, West SG, Aiken LS. 2003 Applied multiple regression/correlation analysis for the behavioral sciences. Third Edition. Mahwah, NJ: Lawrence Erlbaum Associates.

14. R Core Team. 2020 R: A language and environment for statistical computing. R Foundation for Statistical Computing, Vienna, Austria. URL https://www.Rproject.org/.

15. Revelle W. 2020 psych: Procedures for personality and psychological research, Northwestern University, Evanston, Illinois, USA, https://CRAN.Rproject.org/package $=$ psych Version $=2.0 .7$.

16. Jauk E, Benedek M, Dunst B, Neubauer AC. 2013 The relationship between intelligence and creativity: New support for the threshold hypothesis by means of empirical breakpoint detection. Intelligence 41, 212-221. (doi:10.1016/j.intell.2013.03.003)
17. Sorjonen K, Ingre M, Melin B. 2019 Threshold-like associations as a function of disturbance. PeerJ 7 , e7891. (doi:10.7717/peerj.7891)

18. Sorjonen K, Melin B. 2020 Diminishing returns as a function of disturbance. PeerJ 8, e9490. (doi:10.7717/peerj.9490)

19. Larson GE, Alderton DL. 1990 Reaction time variability and intelligence: A "worst performance" analysis of individual differences. Intelligence 14, 309-325. (doi:10.1016/0160-2896(90)90021-K)

20. Schubert A-L. 2019 A meta-analysis of the worst performance rule. Intelligence $\mathbf{7 3}, 88-100$. (doi:10.1016/j.intell.2019.02.003)

21. Coyle TR. 2001 IQ is related to the worst performance rule in a memory task involving children. Intelligence 29, 117-129. (doi:10.1016/S0160-2896(00)00044-1)

22. Coyle TR. 2003 A review of the worst performance rule: Evidence, theory, and alternative hypotheses. Intelligence 31, 567-587. (doi:10.1016/S01602896(03)00054-0)

23. Jensen AR. 1992 The importance of intraindividual variation in reaction time. Personal. Individ. Differ. 13, 869-881.

24. Sorjonen K, Madison G, Melin B, Ullén F. 2020 The Correlation of Sorted Scores Rule. Intelligence $\mathbf{8 0}$, 101454. (doi:10.1016/j.intell.2020.101454)

25. Sorjonen K, Madison G, Hemmingsson T, Melin B, Ullén F. 2021 Further evidence that the worst performance rule is a special case of the correlation of sorted scores rule. Intelligence $\mathbf{8 4}, 101516$. (doi:10.1016/j.intell.2020.101516)

26. Doebler P, Scheffler B. 2016 The relationship of choice reaction time variability and intelligence: A meta-analysis. Learn. Individ. Differ. 52, 157-166. (doi:10.1016/j.lindif.2015.02.009)

27. Jensen AR. 2006 Clocking the mind: mental chronometry and individual differences. 1 st ed. Amsterdam; Boston; London: Elsevier.

28. Eriksson K, Häggström O. 2014 Lord's Paradox in a Continuous Setting and a Regression Artifact in Numerical Cognition Research. PLOS ONE 9, e95949. (doi:10.1371/journal.pone.0095949)

29. Sorjonen K, Melin B, Ingre M. 2019 Predicting the Effect of a Predictor When Controlling for Baseline. Educ. Psychol. Meas. 79, 688-698. (doi:10.1177/0013164418822112)

30. Kahneman D. 1965 Control of spurious association and the reliability of the controlled variable. Psychol. Bull. 64, 326-329. (doi:10.1037/h0022529)

31. Sorjonen K, Melin B, Ingre M. 2020 Accounting for Expected Adjusted Effect. Front. Psychol. 11, 542082. (doi:10.3389/fpsyg.2020.542082) 